Case Report

\title{
Optical Coherence Tomography Angiography of Macular Telangiectasia Type 2 with Associated Subretinal Neovascular Membrane
}

\author{
Victor M. Villegas and Jaclyn L. Kovach \\ Department of Ophthalmology, Bascom Palmer Eye Institute, University of Miami Miller School of Medicine, Miami, FL, USA \\ Correspondence should be addressed to Victor M. Villegas; v.villegas@med.miami.edu
}

Received 27 July 2017; Accepted 22 October 2017; Published 9 November 2017

Academic Editor: Hsin-Yi Chen

Copyright (C) 2017 Victor M. Villegas and Jaclyn L. Kovach. This is an open access article distributed under the Creative Commons Attribution License, which permits unrestricted use, distribution, and reproduction in any medium, provided the original work is properly cited.

\begin{abstract}
Optical coherence tomography angiography (OCTA) is a recently established noninvasive technology for evaluation of the retinal and choroidal vasculature. The literature regarding the findings in macular telangiectasia type 2 (MacTel2) is scarce. We report the OCTA findings associated with a subject with MacTel2 and secondary subretinal neovascularization (SNV). The commercially available Cirrus 5000 with AngioPlex (Zeiss, Jena, Germany) was used, without any subsequent image modification or processing. Subretinal neovascularization was detectable with OCTA at the level of the outer retina and choriocapillaris. Microvascular abnormalities associated with MacTel2 were present mostly in the deep capillary plexus of the retina temporally.
\end{abstract}

\section{Introduction}

Macular telangiectasia type 2 (MacTel2) is a bilateral retinovascular disease that is typically acquired during middle age and may lead to visual loss [1-3]. The hallmark of the disease is retinal vascular ectasia and neural atrophy of the macula [4]. This condition may pose a diagnostic challenge when evaluated with indirect ophthalmoscopy due to the subtle foveal findings. Initially, the only finding on fundus examination could be a decrease in retinal transparency temporal to the fovea. In the early nonproliferative phase of this condition, other clues to diagnosis can be an increase in central foveal autofluorescence related to a reduction in macular pigment. Fluorescein angiography typically demonstrates leakage from abnormal retinal vessels temporal to the fovea. Spectral domain optical coherence tomography (SDOCT) has offered great insight into the pathogenesis of this condition and shows atrophic abnormalities throughout the retinal layers in the foveal including an inner lamellar cyst with internal limiting membrane drape and disruption of the ellipsoid zone. These changes are thought to be due to degeneration of Müller cells and photoreceptors $[5,6]$. Secondary subretinal neovascularization (SNV) can arise in the proliferative phase and form connections with choroidal vessels [7]. The presence of these vessels can be challenging to identify on FA in the setting of temporal foveal leakage.

Optical coherence tomography angiography (OCTA) is a new, fast, noninvasive imaging modality that allows detection of blood flow through the retinal and choroidal plexuses without intravenous dye injection [8]. Various retinal and choroidal diseases have been described using OCTA, including macular telangiectasia [9-20]. Currently, the literature regarding OCTA characteristics of MacTel2 patients with SRNV is limited.

The purpose of this report is to discuss the OCTA features of nonproliferative and proliferative MacTel2 imaged with OCTA. The commercially available Cirrus 5000 with AngioPlex (Zeiss, Jena, Germany) was used, without any subsequent image modification or processing.

\section{Case Report}

A 63-year-old male with history of well-controlled type 2 diabetes mellitus, essential hypertension, and heart disease was referred to our clinic due to visual loss in the right eye (OD). Prior ophthalmic history included radial keratotomy 


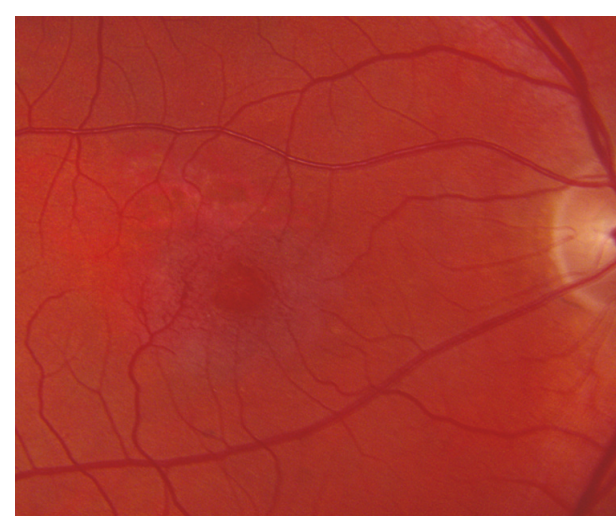

(a)

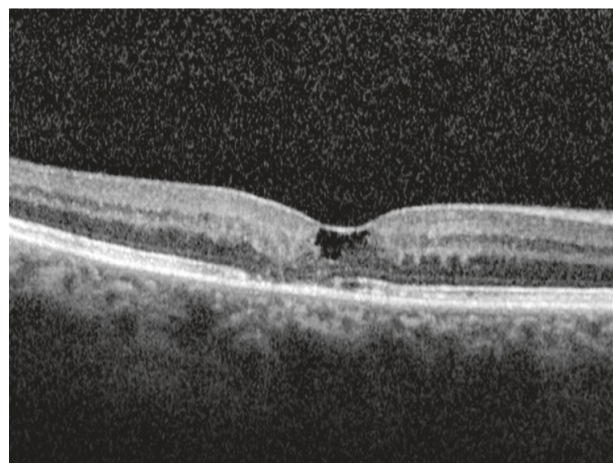

(c)

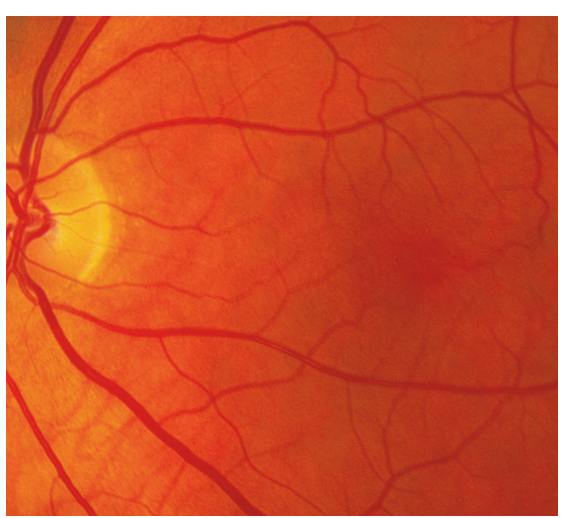

(b)

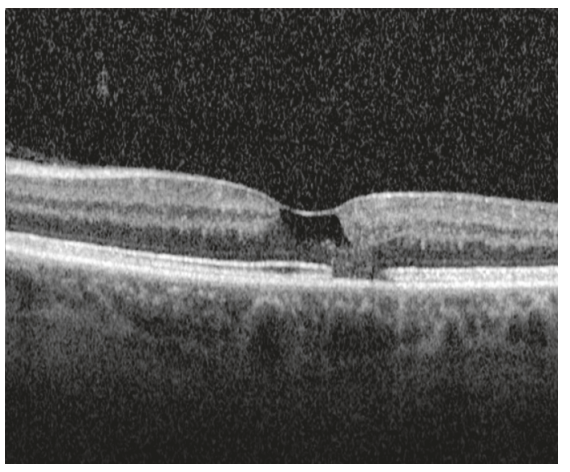

(d)

Figure 1: Macular telangiectasia type 2 initial presentation. (a) Fundus photograph of the right macula shows foveal pigment mottling and changes in the perifoveal vasculature. (b) Fundus photography of the left macula shows foveal pigment mottling. (c) Spectral domain optical coherence tomography (SDOCT) reveals an atrophic cyst and ellipsoid zone disruption in the right macula. (d) Similar SDOCT changes are present in the left macula.

and LASIK in both eyes (OU). He also had uneventful cataract surgery with intraocular lens implantation OD and YAG capsulotomy. Best-corrected visual acuity was 20/50 OD and 20/20 in the left eye (OS). Manifest refraction was -1.00 $+1.50 \times 52$ OD and $+1.50+1.75 \times 42$ OS. Intraocular pressure was $17 \mathrm{mmHg}$ in both eyes (OU). Pupils were equally round and equally reactive to light. Anterior segment examination was remarkable for radial keratotomy with pseudophakia OD and nuclear sclerosis +1 OS. No evidence of intraocular inflammation was seen.

Fundus examination was remarkable for foveal pigment mottling OU. No diabetic or hypertensive retinopathy was present. SDOCT revealed atrophic cysts and ellipsoid zone disruption OU (Figure 1). Given the characteristic SDOCT findings, a diagnosis of macular telangiectasia type 2 was made and the patient was monitored every 6 months. Two years after initial examination, the patient developed acute visual loss to the level of 20/150 and presented with macular hemorrhage OD (Figure 2).

Macular OCTA was performed bilaterally. OCTA OD at the level of the outer retina and choriocapillaris shows subretinal neovascularization (Figure 3). OCTA OS reveals microvascular abnormalities in the deep capillary plexus of the retina most prominent temporally.

\section{Discussion}

This case demonstrates clinical features of nonproliferative and proliferative MacTel2 imaged with OCTA. Early in this condition changes in the retinal microvasculature begin temporal to the fovea in the deep capillary plexus. These abnormalities then extend circumferentially and into the superficial capillary plexus. Anastomoses form between both plexuses and retinal atrophy progresses. This can progress to SRN that can form connections with choroidal vessels [21].

OCTA technology can facilitate diagnosis of all stages of MacTel2 and essentially obviate the need for FA which does not discriminate between superficial and deep retinal vasculature. Also, the detection of subretinal neovascularization in cases in which there is no macular hemorrhage and when temporal leakage on $\mathrm{FA}$ is prominent can become challenging on FA.

MacTel2 etiology continues to be poorly understood. Perifoveal Müller abnormalities may be a common pathway associated with the disease [1,4]. Loss of regulation of these cells may lead to photoreceptor death, dysregulation of angiogenic and inflammatory factors, vascular ectasia, and subsequent subretinal neovascularization [22]. Histopathologic studies have shown thickening of retinal capillaries and 


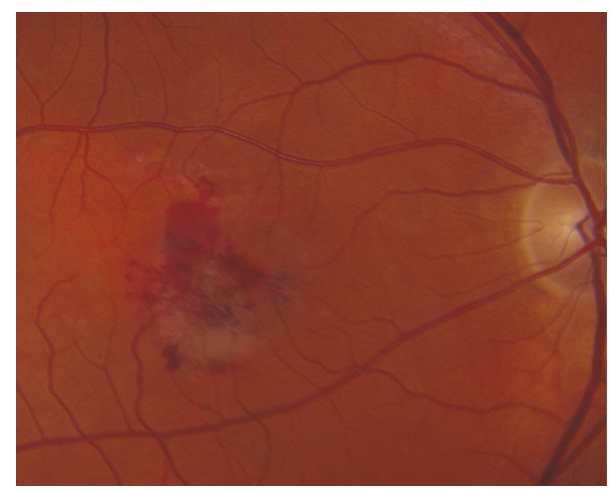

(a)

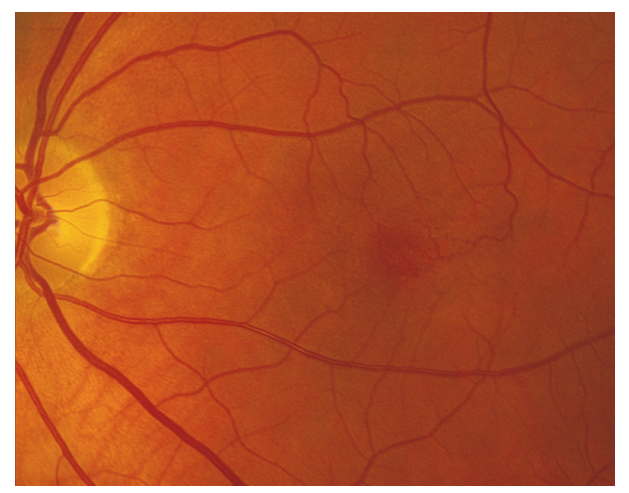

(b)

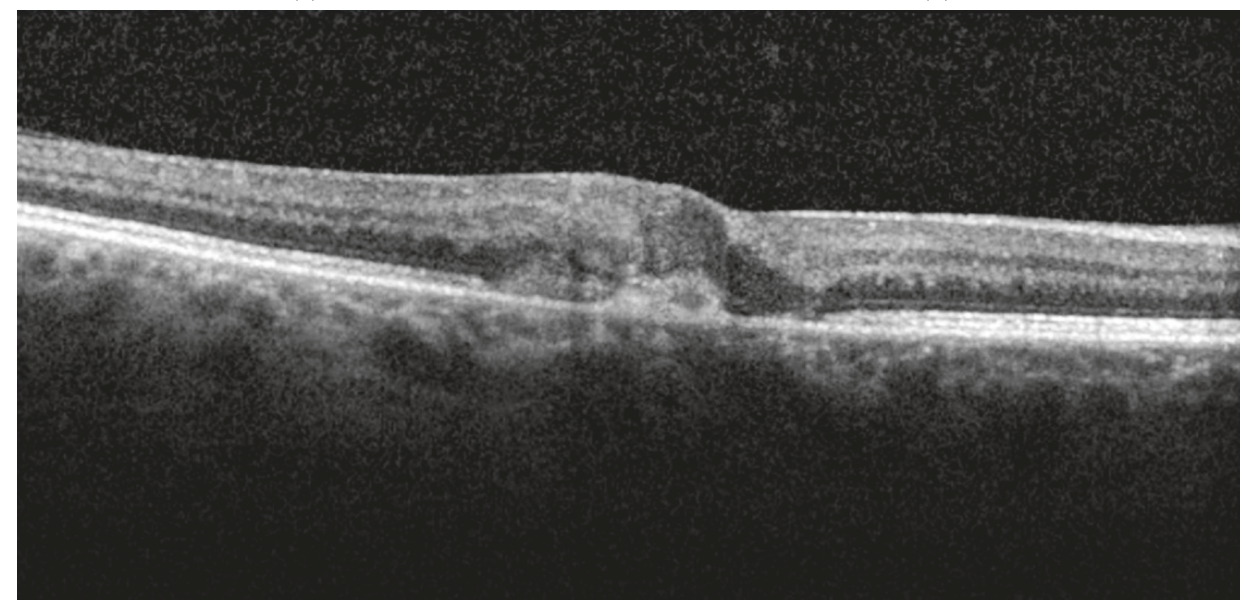

(c)

Figure 2: Fundus photography 2 years after initial presentation. (a) Macular hemorrhages are present in the right eye. (b) Stable foveal pigment mottling is present in the left eye. (c) SDOCT of the right macula reveals a subretinal neovascular membrane.
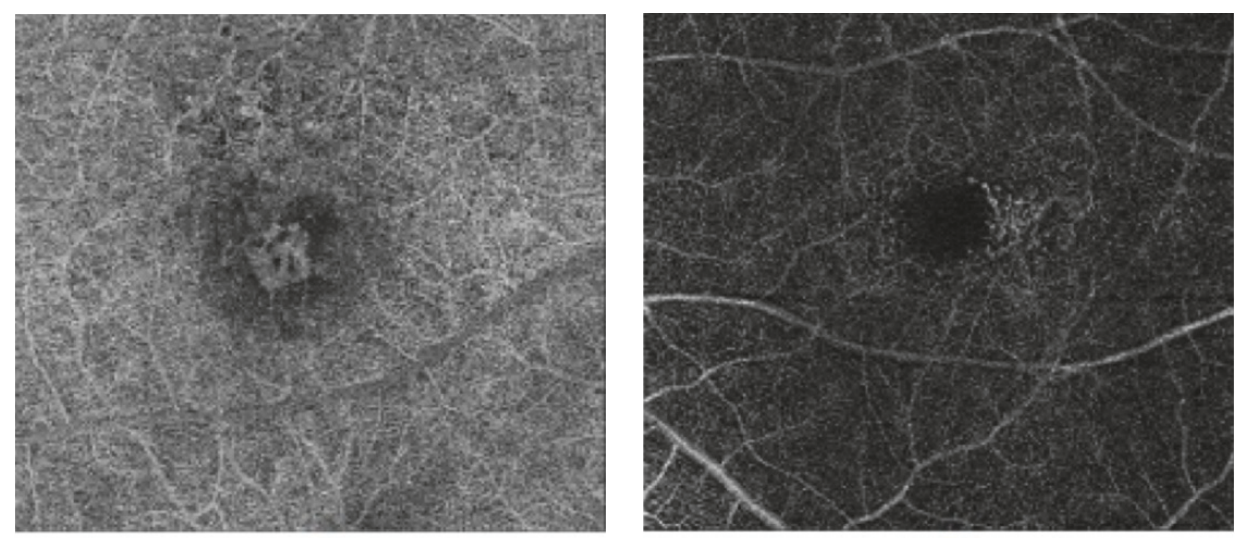

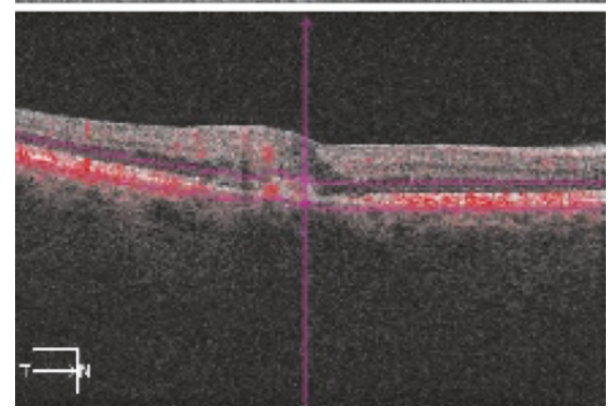

(a)

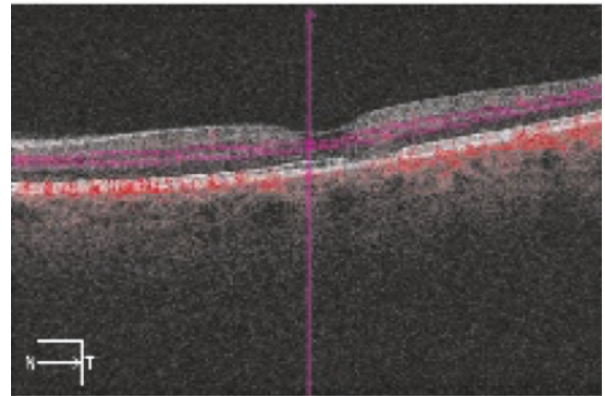

(b)

FIGURE 3: (a) OCTA OD at the level of the outer retina and choriocapillaris shows subretinal neovascularization. (b) OCTA OS demonstrates microvascular abnormalities in the deep capillary plexus of the retina most prominent temporally. 
loss of Müller cells in subjects with macular telangiectasia $[6,23]$. Recent studies have suggested that subjects with diabetes mellitus and hypertension are more likely to have MacTel2 [24]. This may be due to ischemic changes at the level of Müller cells. However, the mechanisms behind systemic disease and MacTel2 remain poorly understood. Further studies will elucidate the mechanisms that explain such associations.

In conclusion, OCTA is a new, fast, noninvasive imaging technology that has enabled improved understanding of the pathophysiology of many retinal vascular diseases including MacTel2. For this condition, OCTA not only facilitates diagnosis but also enables the clinician to monitor disease progression and quantify response to anti-VEGF therapy. Future studies with OCTA will hopefully illuminate additional features of MacTel 2 and provide a better understanding of this complicated disease.

\section{Conflicts of Interest}

The authors declare that they have no conflicts of interest.

\section{Acknowledgments}

This case report is partially supported by NIH Center Core Grant P30EY014801, Research to Prevent Blindness Unrestricted Grant, and the Department of Defense.

\section{References}

[1] J. D. M. Gass and B. A. Blodi, "Idiopathic juxtafoveolar retinal telangiectasis. Update of classification and follow-up study," Ophthalmology, vol. 100, no. 10, pp. 1536-1546, 1993.

[2] L. A. Yannuzzi, A. M. C. Bardal, K. B. Freund, K.-J. Chen, C. M. Eandi, and B. Blodi, "Idiopathic macular telangiectasia," JAMA Ophtalmology, vol. 124, no. 4, pp. 450-460, 2006.

[3] J. L. Kovach and P. J. Rosenfeld, "Bevacizumab (avastin) therapy for idiopathic macular telangiectasia type II," Retina, vol. 29, no. 1, pp. 27-32, 2009.

[4] M. B. Powner, M. C. Gillies, M. Tretiach et al., "Perifoveal müller cell depletion in a case of macular telangiectasia type 2," Ophthalmology, vol. 117, no. 12, pp. 2407-2416, 2010.

[5] S. M. Cohen, M. L. Cohen, F. El-Jabali, and S. E. Pautler, "Optical coherence tomography findings in nonproliferative group $2 \mathrm{~A}$ idiopathic juxtafoveal retinal telangiectasis," Retina, vol. 27, no. 1, pp. 59-66, 2007.

[6] J. D. M. Gass and W. R. Green, "Histopathologic study of presumed parafoveal telangiectasis [2] (multiple letters)," Retina, vol. 20, no. 2, pp. 226-227, 2000.

[7] M. J. Potter, S. M. Szabo, E. Y. Chan, and A. H. C. Morris, "Photodynamic therapy of a subretinal neovascular membrane in type 2A idiopathic juxtafoveolar retinal telangiectasis," American Journal of Ophthalmology, vol. 133, no. 1, pp. 149-151, 2002.

[8] Y. Jia, O. Tan, J. Tokayer et al., "Split-spectrum amplitudedecorrelation angiography with optical coherence tomography," Optics Express, vol. 20, no. 4, pp. 4710-4725, 2012.

[9] N. Takase, M. Nozaki, A. Kato, H. Ozeki, M. Yoshida, and Y. Ogura, "Enlargement of foveal avascular zone in diabetic eyes evaluated by en face optical coherence tomography angiography," Retina, vol. 35, no. 11, pp. 2377-2383, 2015.
[10] N. Suzuki, Y. Hirano, M. Yoshida et al., "Microvascular abnormalities on optical coherence tomography angiography in macular edema associated with branch retinal vein occlusion," American Journal of Ophthalmology, vol. 161, pp. 126-132el, 2016.

[11] F. Coscas, A. Glacet-Bernard, A. Miere et al., "Optical coherence tomography angiography in retinal vein occlusion: evaluation of superficial and deep capillary plexa," American Journal of Ophthalmology, vol. 161, pp. 160-171e2, 2016.

[12] K. Sambhav, S. Grover, and K. V. Chalam, "The application of optical coherence tomography angiography in retinal diseases," Survey of Ophthalmology, 2017.

[13] M. K. Durbin, L. An, N. D. Shemonski et al., "Quantification of Retinal Microvascular Density in Optical Coherence Tomographic Angiography Images in Diabetic Retinopathy," JAMA Ophthalmology, vol. 135, no. 4, p. 370, 2017.

[14] N. A. Yannuzzi, N. Z. Gregori, L. Roisman, N. Gupta, B. E. Goldhagen, and R. Goldhardt, "Fluorescein angiography versus optical coherence tomography angiography in macular telangiectasia type i treated with bevacizumab therapy," Ophthalmic Surgery, Lasers \& Imaging Retina, vol. 48, no. 3, pp. 263-266, 2017.

[15] S. G. Schwartz and J. W. Harbour, "Multimodal Imaging of Astrocytic Hamartomas Associated With Tuberous Sclerosis," Ophthalmic Surgery, Lasers \& Imaging Retina, vol. 48, no. 9, pp. 756-758, 2017.

[16] S. Y. Chan, Q. Wang, Y. X. Wang, X. H. Shi, J. B. Jonas, and W. B. Wei, "Polypoidal choroidal vasculopathy upon optical coherence tomographic angiography," Retina, p. 1, 2017.

[17] M. Pellegrini, F. Corvi, E. A. Say, C. L. Shields, and G. Staurenghi, "Optical coherence tomography angiography features of choroidal neovascularization associated with choroidal nevus," Retina, p. 1, 2017.

[18] M. A. Gonzalez, D. Shechtman, J. M. Haynie, and L. Semes, "Unveiling idiopathic macular telangiectasia: clinical applications of optical coherence tomography angiography," European Journal of Ophthalmology, vol. 27, no. 4, pp. 0-0, 2017.

[19] R. F. Spaide, J. M. Klancnik Jr., and M. J. Cooney, "Retinal vascular layers imaged by fluorescein angiography and optical coherence tomography angiography," JAMA Ophthalmology, vol. 33, no. 1, pp. 45-50, 2015.

[20] L. Chidambara, S. G. K. Gadde, N. K. Yadav et al., "Characteristics and quantification of vascular changes in macular telangiectasia type 2 on optical coherence tomography angiography," British Journal of Ophthalmology, vol. 100, no. 11, pp. 1482-1488, 2016.

[21] L. Roisman and P. J. Rosenfeld, "Optical Coherence Tomography Angiography of Macular Telangiectasia Type 2," Developments in Ophthalmology, vol. 56, pp. 146-158, 2016.

[22] W. Shen, M. Fruttiger, L. Zhu et al., "Conditional müller cell ablation causes independent neuronal and vascular pathologies in a novel transgenic model," The Journal of Neuroscience, vol. 32, no. 45, pp. 15715-15727, 2012.

[23] W. R. Green, H. A. Quigley, Z. de la Cruz, and B. Cohen, "Parafoveal retinal telangiectasis," Retina, vol. 25, no. Supplement, pp. 162-170, 2005.

[24] T. E. Clemons, M. C. Gillies, E. Y. Chew et al., "Medical characteristics of patients with macular telangiectasia type 2 (MacTel Type 2) MacTel Project Report No. 3," Ophthalmic Epidemiology, vol. 20, no. 2, pp. 109-113, 2013. 


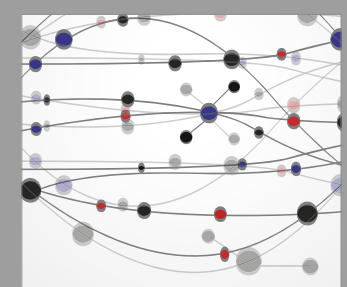

The Scientific World Journal
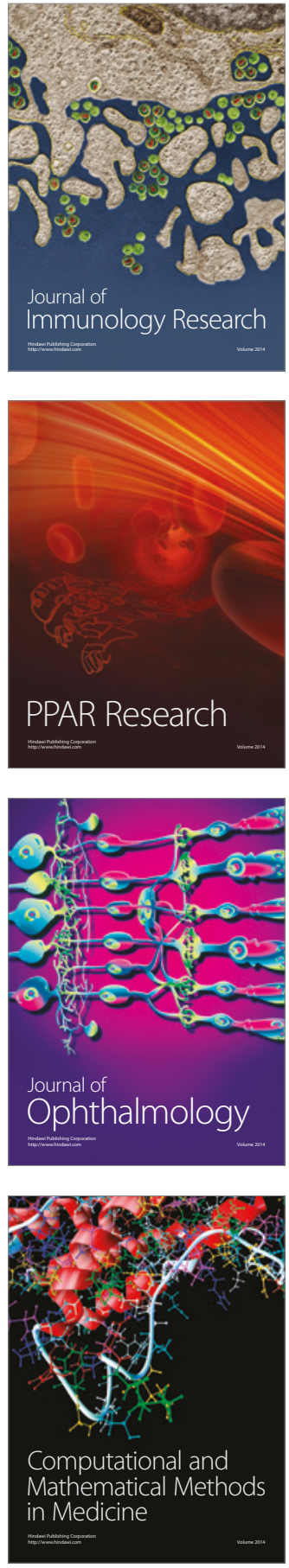

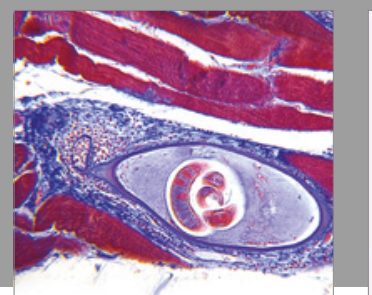

Gastroenterology Research and Practice
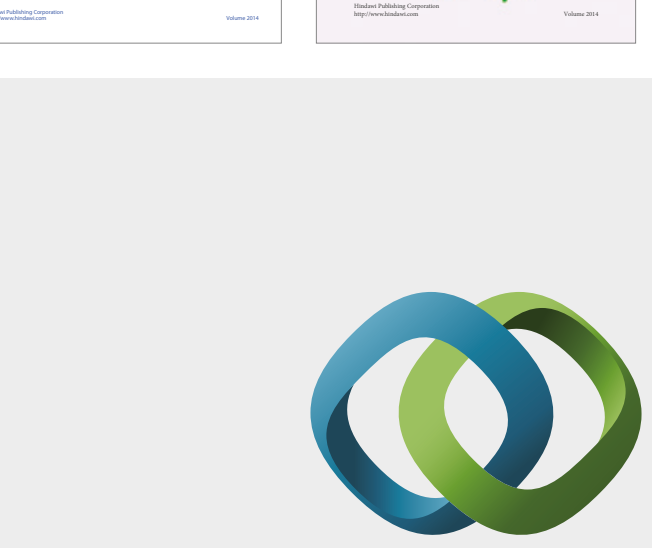

\section{Hindawi}

Submit your manuscripts at

https://www.hindawi.com
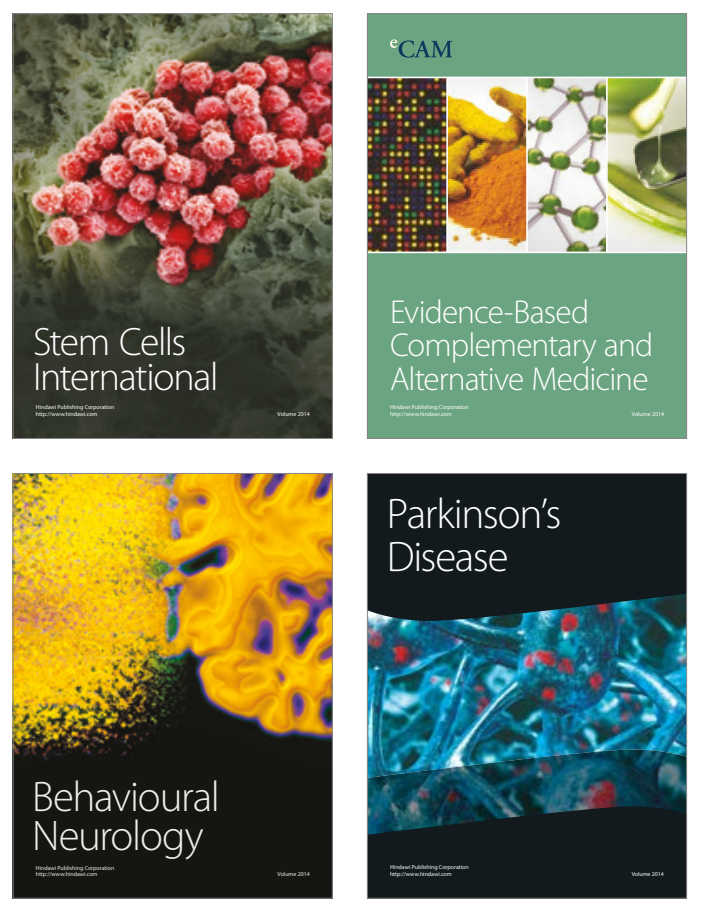
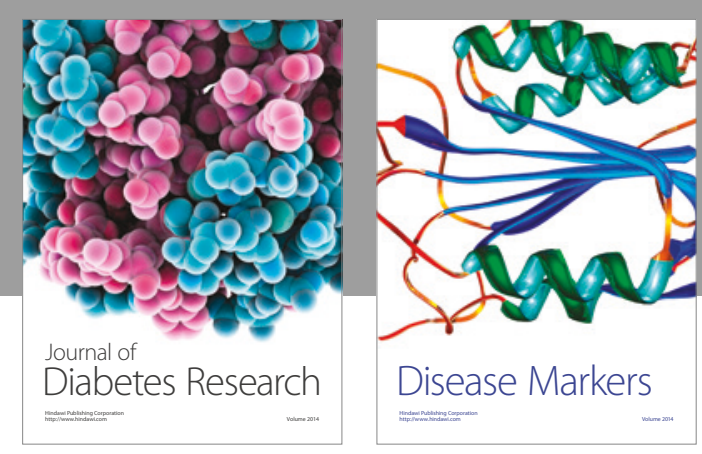

Disease Markers
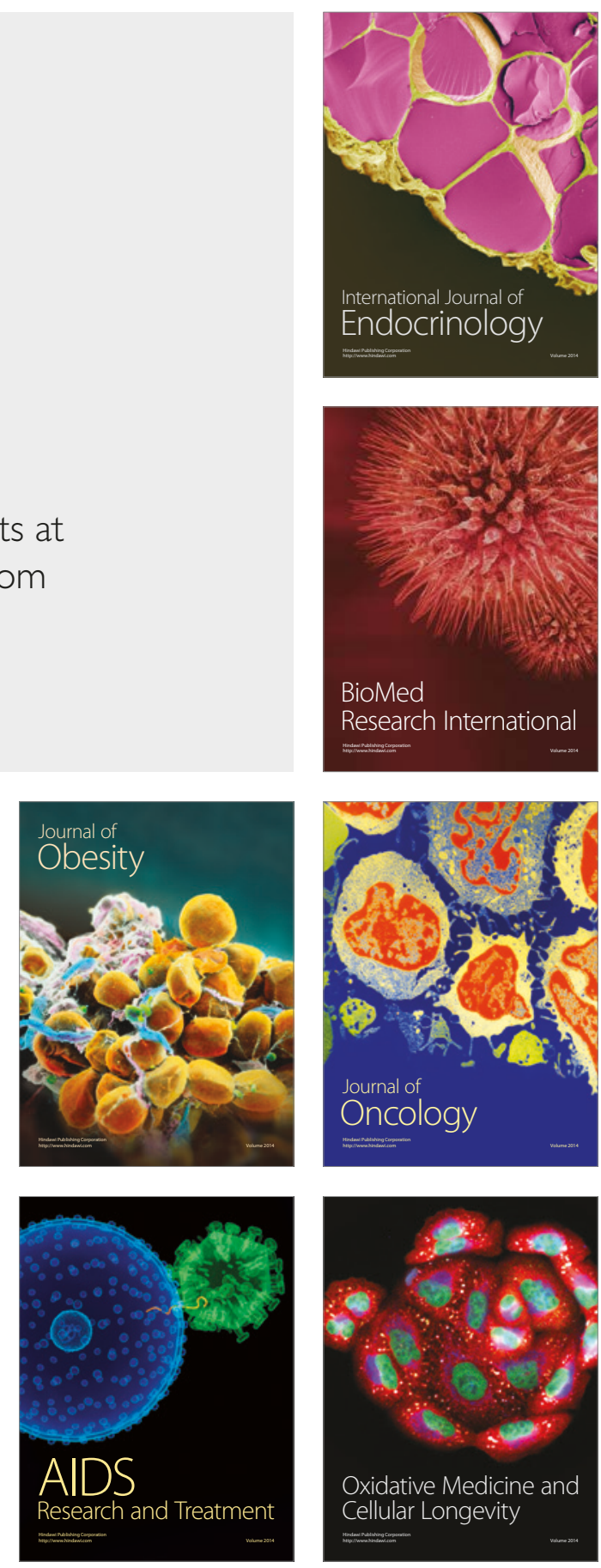